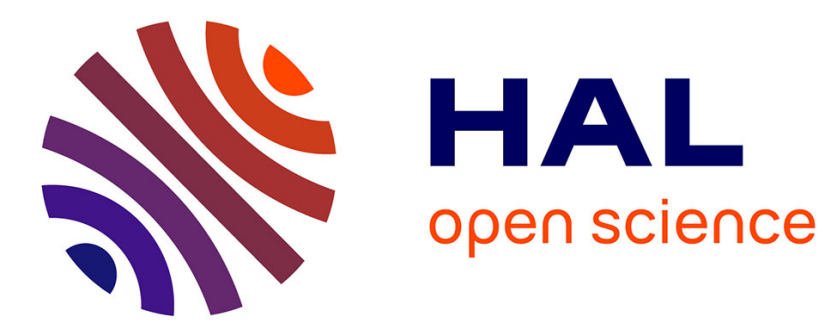

\title{
Du champ migratoire aux circulations : une lecture des migrations internationales
}

\author{
Mohamed Kamel Doraï, Marie-Antoinette Hily
}

\section{To cite this version:}

Mohamed Kamel Doraï, Marie-Antoinette Hily. Du champ migratoire aux circulations: une lecture des migrations internationales. Géographes associés, 2005, 29, pp.19-26. halshs-00687528

\section{HAL Id: halshs-00687528 \\ https://shs.hal.science/halshs-00687528}

Submitted on 13 Apr 2012

HAL is a multi-disciplinary open access archive for the deposit and dissemination of scientific research documents, whether they are published or not. The documents may come from teaching and research institutions in France or abroad, or from public or private research centers.
L'archive ouverte pluridisciplinaire HAL, est destinée au dépôt et à la diffusion de documents scientifiques de niveau recherche, publiés ou non, émanant des établissements d'enseignement et de recherche français ou étrangers, des laboratoires publics ou privés. 


\title{
DU CHAMP MIGRATOIRE AUX CIRCULATIONS: UNE LECTURE DES MIGRATIONS INTERNATIONALES
}

\author{
Kamel DORAÏ ${ }^{1}$, Marie-Antoinette HILY ${ }^{2}$
}

La mondialisation des migrations induit trois phénomènes principaux: le passage du retour définitif au retour alternatif, le développement des diasporas et des réseaux communautaires et une circulation accrue des individus. Ils interrogent la représentation de l'homogénéité de la nation et notamment l'adéquation entre Etat, nation et territoire dans un monde caractérisé par l'interdépendance accrue, les processus de délocalisation, les identités multiples.

Dès lors, se pose la question de la gestion politique de ces modes d'organisation. Ces processus soulèvent en effet des interrogations relatives aux circulations transnationales et au statut juridique des migrants, en de tous autres termes que ceux des années soixante-dix, qui opposaient arrêt ou reprise de l'immigration, intégration ou retour. Enfin, ils caractérisent des dispersions qui ne sont ni passagères ni ponctuelles mais donnent lieu à des stratégies où l'éclatement dans l'espace est lui-même utilisé comme ressource (Ma Mung, 1999).

Successivement et depuis les années 50 les chercheurs ont forgé un ensemble de concepts: noria, champ migratoire ou espace migratoire, filières, circulation migratoire pour rendre compte du sens des déplacements de main-d'oeuvre "étrangère" et des modes d'organisation d'immigrants. Ces concepts et leurs champs d'application traduisent l'évolution tant des phénomènes migratoires au cours de l'histoire que la façon dont les chercheurs tentent de les analyser et d'en donner une interprétation.

\section{De la noria au champ migratoire}

\subsection{Norias simples et complexes}

Robert Montagne (1954) est probablement le premier à utiliser le terme de "noria" humaine pour désigner l'arrivée de migrants d'un village d'origine en remplacement de ceux rentrés définitivement. Ce terme peu utilisé dans les travaux ultérieurs sur les migrations a été largement critiqué notamment par Abdelmalek Sayad (1977). Le terme de "noria complexifiée" sera cependant repris par P. Gonin (1997) pour décrire la diversification croissante des échanges entre les villages de la vallée du fleuve Sénégal et la France.

\footnotetext{
${ }^{1}$ Chargé de recherche CNRS, MIGRINTER - Université de Poitiers / CNRS : UMR 6588

${ }^{2}$ Chargée de recherche CNRS, MIGRINTER - Université de Poitiers / CNRS : UMR 6588
} 
Patrick Gonin (1997), dans son travail sur la circulation migratoire entre la France et la vallée du fleuve Sénégal, étudie le passage de la noria simple à la noria complexe. La "première noria traduit les mécanismes d'échanges d'un lieu à un autre, régis globalement par les systèmes de dépendances" (p. 142). Ce système fonctionne avec une durée de résidence courte en France, le migrant étant remplacé rapidement par une nouvelle personne, dans le contexte des frontières ouvertes d'avant 1974. Avec la fermeture des frontières et le regroupement familial, les immigrés vont s'installer en France. Les rapports avec le village d'origine se modifient progressivement. Les départs chutent avec la fermeture des frontières et l'arrêt de l'immigration. De nouvelles pratiques se développent entre pays d'origine et pays d'accueil que P. Gonin peut résumer ainsi :

"Les flux circulants entre pays de départ et pays d'arrivée se sont fortement amplifiés, ils ne sont plus le fait de personnes, certainement même moins pour les devises; ils sont devenus immatériels" (Gonin, p. 252). Les nouveaux migrants se dirigent maintenant vers des régions plus proches. "Ainsi le contenu de ce qui circule change: ici c'est une proposition de soutien de la conception d'une nouvelle réalisation, là des démarches techniques sur les différentes caractéristiques des groupes motopompes, ailleurs de la formation pour mener à bien un pro/et..." (Gonin, p. 252). L'auteur souligne, dans l'exemple qu'il traite, que la complexification de la noria aboutit à la constitution de deux groupes distincts chez les migrants originaires de la vallée du fleuve Sénégal. Un premier groupe décide de s'installer dans un des pays, ou en France (très minoritaire) ou dans son pays d'origine. Un second groupe "cherche quant à lui à pénétrer un peu plus la société d'installation, s'insère dans des réseaux associatifs, politiques et syndicaux, et il utilisera cette connaissance acquise pour donner un autre contenu à la circulation migratoire" (Gonin, p.305).

\subsection{Champ et filière migratoire}

Le concept de champ migratoire est né dans un contexte scientifique où les théories et les méthodes de la géographie classique ne "rendaient pas suffisamment compte des rapports à 1'espace, de ceux qui se nouent entre le migrant et les espaces pratiqués dans le cadre de parcours migratoires souvent complexes, qu'il s'agisse de lieux de départ, d'arrivée, d'implantation ou d'installation mais aussi des lieux de passage, de transit, et peut-être même plus tard des espaces de rebondissement siège de nouvelles migrations" (Simon, 2000).

Concept né dans la géographie quantitative suédoise des années 1960, repris par Courgeau, socio-démographe à l'INED, en 1970, puis utilisé par les géographes qui traitent des migrations et dont une des préoccupations concerne la traduction spatiale des relations migratoires. Il permet de saisir à la fois les aires de départ et d'arrivée et des comportements (flux, organisations, services ... ). Concernant l'analyse de l'espace relationnel des Tunisois, Gildas Simon définit le champ migratoire comme: "l'ensemble de l'espace parcouru, pratiqué par les migrants. La notion faisant référence à un espace spécifique, structuré par des flux importants, significatifs; appliqué au domaine international, elle englobe à la fois le pays de départ et le pays d'emploi".

C'est un véritable espace humain, fortement structuré et dynamique. [Le champ migratoire] aire d'extension des migrations d'une population donnée, à l'image d'un champ magnétique, est un espace dans lequel la dite population construit un ou des réseaux de relations". Le 
champ migratoire se constitue à partir de migrations d'individus organisées en filières migratoires.

Les filières migratoires sont abordées comme facteur d'organisation d'un espace relationnel: le concept de filières migratoires s'est construit dans les recherches françaises en s'inspirant de travaux géographiques sur les migrations intra-nationales et les relations villes-campagnes (Schwab, 1971). Béteille (1974) montrera que des filières migratoires remontant au milieu du $19^{\text {ème }}$ siècle avaient "non seulement projeté les Aveyronnais dans un espace de dimension nationale, voire internationale, mais surtout abouti à l'organisation d'un véritable espace humain et relationnel'. G. Simon (1979), et M. Poinard (1991) appliqueront une démarche et une conceptualisation similaires pour mettre en évidence, l'un dans son analyse de l'espace des travailleurs tunisiens en France, l'autre à propos de l'émigration des Portugais, la constitution d'un "champ social international' mettant en jeu des relations préférentielles, des filières, des comportements socioculturels et des intérêts économiques.

\section{Du va-et-vient à la circulation}

L'organisation du champ migratoire et la mondialisation des migrations se traduisent par le développement des pratiques de va-et-vient ou de circulation migratoire. À la différence du terme de noria, celui de "va-et-vient" (Charbit, Hily, Poinard, 1997) a connu un meilleur destin. Initialement il apparaît dans les travaux de R. Montagne consacrés aux travailleurs kabyles. La notion de va-et-vient qui sous-tend celle de circulation migratoire n'est donc pas récente, elle préfigure déjà le fonctionnement en réseau des groupes migrants et leur capacité de maintenir des liens entre pays d'origine et pays d'accueil.

L'expression "circulation migratoire" apparaît dans les années 80 pour signifier des phénomènes de mobilité qui ne peuvent plus être décrits uniquement dans le cadre de relations entre deux pays ou États-nations (pays d'origine et pays d'installation). Cette notion tente de saisir la complexité des phénomènes liés à la migration et à l'expérience postmigratoire, ainsi que la vision statique des migrations comme mouvement "entre deux sédentarités" (Tarrius, 1992), ainsi que la dichotomie existant dans les recherches sur les migrations internationales entre celles portant sur les sociétés d'installation organisées autour des problématiques de l'intégration et celles s'intéressant aux pays d'origine centrées sur des problématiques de développement. L'objectif est d'avoir une approche globale, et non plus dichotomique, des migrations. Il s'élargit progressivement et vise à prendre en compte tout à la fois l'ensemble des espaces concernés par les migrations, les déplacements accrus des personnes entre lieux d'origine et d'arrivée (les va-et-vient) et les flux matériels (biens, services, remises) et idéels (normes, valeurs, représentations) induits par les migrations. En conséquence c'est le migrant plutôt que l'immigré ou l'émigré qui est au centre de l'analyse, l'accent est mis sur les pratiques et sur les initiatives des personnes plutôt que sur les déterminants de la migration. Avec des notions connexes comme celles de territoires circulatoires, de réseaux transnationaux, de diasporas ainsi que celle de transnationalisme qui dans la littérature scientifique anglophone essaie de prendre en compte les mêmes phénomènes, l'intérêt théorique et empirique de la notion de circulation migratoire conduit à réinterpréter le champ d'expérience des migrants et permet de reposer à nouveaux frais la question des temporalités sociales, des rapports des hommes à l'espace et à leur histoire identitaire. 
Dans cette perspective, tout un courant de recherche, en France notamment, va produire une réflexion théorique originale et développer des travaux empiriques sur les mobilités des populations. Le recadrage de la problématique sur les migrations et la modification de ses objets de recherches s'inscrit dans le déplacement du débat de la sociologie de l'intégration vers une sociologie de la constitution de savoir-faire migratoires et de la construction de réseaux transnationaux producteurs de richesse économique.

\section{L'émergence d'un espace transnational}

Douglas Massey (1988), qui étudie les migrations à la frontière Etats-Unis / Mexique, définit le réseau migratoire comme «l'ensemble des liens interpersonnels qui relient les migrants, les futurs migrants, et les non-migrants dans les espaces d'origines et de destination, à travers les liens de parenté, d'amitié, et une origine communautaire partagée". Isabelle TaboadaLeonetti et alii (1994) dans une étude sur la communauté chinoise d'Ile-de-France montre que "l'analyse des structures des relations des migrants en termes de réseau a l'avantage de dépasser le dualisme "relations avec les compatriotes/ relations avec le pays d'accueil" qui est le propre des études classiques sur l'immigration ».

L'étude des modalités de la circulation migratoire s'inscrit dans un champ d'investigation situé au carrefour de plusieurs disciplines, qui tente de rompre avec des dichotomies: départarrivée, installation-retour, temporaire-permanent, etc. Ce concept, écrit Gildas Simon, différent de celui de migration, fait référence à la mobilité physique des hommes, avec leur itinéraire, leur moyen de transport et la pratique effective et affective de l'espace parcouru. Peu à peu la notion de circulation migratoire ne recouvre plus seulement un certain type de migrations de personnes. Elle concerne également l'ensemble des flux matériels et idéels générés par la circulation des hommes.

Depuis une dizaine d'années se développe dans les sciences sociales une sociologie des réseaux transnationaux, notamment en France et au Royaume-Uni. Ces formes d'organisations sociales basées sur des appartenances multiples obligent les chercheurs à revisiter les notions qui jusqu'à présent servaient à analyser les phénomènes migratoires. Jusqu'à la fin des années 1960, le schéma des migrations tournantes prédominait. Dans les années 1970 (arrêt de l'immigration officielle de travail, politique appropriée de rassemblement des familles, tentative de promouvoir les retours) l'Etat français notamment tente de stabiliser le volume existant de main-d'œuvre immigrée en favorisant les mesures d'intégration. En misant sur les capacités de substitution que représente la "deuxième génération", on s'acheminait vers un système qui tentait de stopper les ressources de mobilité internationale. En d'autres termes les migrants étaient soit "ici" soit "là-bas", rentrés définitivement, pensait-on. A partir des années 1980-1990, les phénomènes de circulation migratoire se sont accentués et complexifiés; en droit avec les zones de libre circulation dont l'Espace Economique Européen, en droit aussi avec l'extension de la durée de validité des titres de séjour et en fait avec la constitution de configurations diasporiques.

Dès lors et comme le souligne A. Tarrius, il s'agit de privilégier dans l'analyse le couple migration/ territoire qui selon lui "fait autrement sens" que le couple immigration/insertion. Pour l'auteur le terme "immigré" appelle "insertion" alors que le "migrant" "est plutôt accolé à "territoire". En posant le problème en ces termes, la recherche se déplace d'un paradigme d'intégration (sous-entendu à l'État-nation) vers un paradigme "mobilitaire". Dans cette 
perspective, la notion de " territoire circulatoire" est le résultat de " ... productions de mémoires collectives et de pratiques d'échanges sans cesse plus amples, où valeurs éthiques et économiques spécifiques créent une culture" (Tarrius, 1994). S. de Tapia (1998) reprend à son compte cette démarche et précise que ce terme a été conçu "comme le support matériel dynamique de plusieurs concepts géographiques et sociologiques de l'étude des migrations internationales ... ; tels que le commerce ethnique (à partir de la notion américaine d'ethnic business), les processus de maintien et de transformation des identités face à ceux de l'intégration et de l'assimilation, les notions d'espace et de champ, de filières et de réseaux migratoires, de circulation, de réseaux sociaux transnationaux, de diaspora".

On précisera que dans la littérature anglophone/nord américaine l'équivalent de la notion de circulation migratoire - - migratory circulation - est peu utilisée. On lui préfère celles de circular migration ou de circulatory migration, qui insistent sur la dimension de migration plutôt que sur celle de circulation. C'est la notion de transnationalisme qui se rapproche le plus de ce que, en France, on désigne par circulation migratoire.

\section{La circulation migratoire : essais de typologies}

La littérature concernant la circulation migratoire nous permet de distinguer deux processus qui la favorisent et l'entretiennent. Le premier est l'installation définitive de groupes migrants dans leurs pays d'accueil. Elle entraîne un va-et-vient entre pays de résidence et pays d'origine lors des vacances ou des visites pluri-annuelles. La circulation est alors le résultat d'une migration de travail "classique". Le second repose sur le développement de la mobilité lié à la circulation entre les différents pôles d'une diaspora ou à des migrations temporaires répétées dans le cadre de contrat de travail à l'étranger à durée déterminée, comme pour les cadres.

Cette distinction nous permet d'élaborer une typologie des circulations migratoires, qui permet de recenser des populations très diverses qui pratiquent des mobilités.

- Les travailleurs migrants "sédentarisés" dans leur pays d'accueil, qui pratiquent un vaet-vient, entre leur lieu de résidence "permanente" et leur pays d'origine (exemples: Turcs, Portugais, Sénégalais ... ).

- Les travailleurs hautement qualifiés qui s'expatrient temporairement à l'étranger dans le cadre de contrats de travail spécifiques, ou qui circulent entre les grandes capitales européennes (exemple: les cadres travaillant dans la finance, ou les ingénieurs qui travaillent dans le Golfe persique).

- Les membres de diaspora qui circulent entre les différents pôles de la diaspora en fonction de la situation politique, législative ou économique (exemples: entrepreneurs chinois ou libanais).

- Les accompagnateurs de marchandises, qui sont des acteurs de la circulation migratoire. Cette fonction tend à se développer du fait de l'interconnexion croissante des économies européennes.

Une typologie peut aussi se baser sur le type de mobilité qu'entraîne la circulation migratoire. On aurait d'un côté des mobilités "lourdes" qui s'accompagnent de forts changements dans la vie du migrant ou qui nécessitent la mobilisation de nombreux acteurs (comme la circulation de biens ou de marchandises qui mobilisent des acteurs dans le domaine du commerce, du transport, des prestations de services divers - assurances, prêts d'argent ... - tant dans le pays 
de départ que le pays d'arrivée) et de l'autre des mobilités "légères" qui se traduisent par de brefs voyages du migrant (vacances, visites familiales ... ).

\section{Conclusion.}

Sous le terme englobant de circulation migratoire, il s'agit pour les chercheurs d'intégrer dans les analyses ce qu'ils considèrent comme des nouvelles formes de mobilités où les migrants semblent s'émanciper des normes du salariat pour s'inscrire dans des activités économiques formelles et informelles portées par des réseaux socio-économiques plus ou moins structurés. Ce sont alors ces dispositifs commerciaux opérants sur des espaces transnationaux qui deviennent centraux dans l'analyse. Ces formes de circulation participent à des dérégulations du marché du travail. Dès lors la question de l'intégration du point de vue des politiques migratoires, est confrontée à une multiplicité des figures de migrants: entrepreneurs, commerçants, aventuriers, vendeurs ambulants, etc.. ; figures qui sont aussi de plus en plus féminines comme celles par exemple des Nana-Benz.

Dans ce contexte, la notion de "projet migratoire" devient elle aussi centrale pour comprendre les logiques d'installation et de mobilité. Elle permet de réinterpréter le champ d'expérience des migrants.

Ces nouveaux questionnements sur les migrations et la modification de ses objets de recherches s'inscrit dans le déplacement du débat de la sociologie de l'intégration vers une sociologie de la constitution de savoir-faire migratoires. Elle implique de centrer les approches vers la production de réseaux créateurs de nouvelles structures de liaisons horizontales et associatives et générateurs de nouvelles solidarités. Il ressort par exemple que la constitution de diasporas ne se mesure pas uniquement en termes de mobilités migratoires. Elles peuvent organiser des groupes, produire de nouveaux rapports à la citoyenneté, renforcer des solidarités propices à lutter contre la précarité et l'incertitude. Elles créent aussi des expériences et des pratiques de contournement des dispositifs juridico-étatiques.

Les modalités contemporaines de la circulation migratoire mettent en évidence le lien problématique entre territorialité et mobilité. La sédentarisation des populations immigrées s'est paradoxalement accompagnée de la mise en place de réseaux sociaux et économiques plus ou moins denses selon les groupes, qui constituent des ressources pour les membres, supports de nouveaux rapports sociaux. Ces formes d'organisations sociales relativement autonomes se basent sur la multipolarité de la migration et l'interpolarité des relations dans un espace extra-territorial (Ma Mung, 1994). On passe ainsi de rapports entre nations à la création d'espaces transnationaux propices à de nouvelles articulations entre identité et territoire.

L'interrelation de plus en plus grande peut à terme amplifier et élargir la circulation migratoire par la densification des échanges dans l'espace européen. Les effets sur les mobilités sont encore sous-estimés, d'autant que le développement de certains pays du tiers monde peut modifier et accélérer la mobilité alternative. Notamment parce qu'une économie qui se développe en se basant sur l'exportation accélère son intégration à l'économie mondiale et augmente son interdépendance avec les autres pays : développement ne signifiant pas automatiquement réduction de la pression migratoire. De surcroît les crises économiques en 
bouleversant les systèmes migratoires peuvent, elles aussi, renforcer et/ou reconfigurer la circulation migratoire.

Dès lors c'est aux temporalités sociales, aux rapports des hommes à l'espace et à leur histoire identitaire qu'il faut revenir si l'on veut saisir les changements sociaux en cours. C'est l'émergence de ces nouvelles configurations migratoires où la circulation tient une place importante qu'il convient d'explorer en promouvant des recherches articulant les approches quantitatives et anthropologiques.

\section{Bibliographie.}

BETEILLE R. (1974) Les Aveyronnais. Essai géographique sur l'espace humain. Poitiers, R. Béteille, Imprimerie l'Union, 573 p.

BETEILLE, Roger (1981) Une nouvelle approche géographique des faits migratoires: champs, relations, espaces relationnels. L'Espace géographique, $\mathrm{n}^{\circ} 3$, pp. 189-197.

CHARBIT Yves, HILY Marie-Antoinette, POINARD Michel (1997) Le va-et-vient identitaire. Migrants Portugais et villages d'origine, Paris, PUF, INED (Cahiers de l'Ined $\left.\mathrm{n}^{\circ} 140\right), 144 \mathrm{p}$.

COURGEAU Bernard (1970) Les champs migratoires en France, Paris, PUF, 158 p.

GONIN Patrick (1997) D'entre deux territoires. Circulations migratoires et développement entre le bassin du fleuve Sénégal et la France, Université des Sciences et Technologies de Lille, Habilitation à Diriger des Recherches, Université de Lille, $384 \mathrm{p}$.

GUILLON, Michelle; MA MUNG, Emmanuel; TABOADA-LEONETTI, Isabelle (1994) Réseaux locaux, réseaux transnationaux des communautés d'origine immigrée en France. Le cas des populations d'origine chinoise dans la région parisienne. Rapport de recherche pour la DATAR, Appel d'offres «Prospective et territoire». Groupe de sociologie du travail, CNRS université Paris 7, 188 p.

MA MUNG Emmanuel (1994) Non-lieu et utopie: la diaspora chinoise et le territoire, L'Espace Géographique, Vol. 1994, n².

MA MUNG Emmanuel, dir. (1996) Mobilités et investissements des émigrés, Maroc, Tunisie, Turquie, Sénégal, Paris, L'Harmattan, Paris, 272 p.

MA MUNG Emmanuel (1999) La dispersion comme ressource, Cultures et Conflits, n³3-34, pp. 89-103.

MASSEY Douglas S. (1988) Theories of International Migration: a Review and Apraisal. Population and Development Review, 19 n³, pp. 431-466.

MONTAGNE Robert (1954) Etude sociologique de la migration des travailleurs musulmans d'Algérie en France. Cahier liminaire, in Robert MONTAGNE (dir.) Etude sociologique de la migration des travailleurs musulmans d'Algérie en France (Cahier liminaire, cahier nO 1 à 8), pp. 3-33.

POINARD Michel (1991) Les Portugais dans l'Emigration: une Géographie de l'Absence, Toulouse - Le Mirail, Thèse pour le Doctorat d'Etat, Géographie et Aménagement, $630 \mathrm{p}$. 
SAYAD Abdelmalek (1977) Les trois « âges» de l'immigration algérienne en France. Actes de la recherche en sciences sociales, juin, pp. 60-79.

SIMON Gildas (1979) L 'Espace des Travailleurs Tunisiens en France (structures et fonctionnement d'un champ migratoire international), Thèse d'Etat de Géographie, Université de Poitiers, éd. G. Simon, 426 p.

SIMON Gildas (1990) Les diasporas maghrébines et la construction européenne. Revue Européenne des Migrations Internationales, 6, n² 2, pp. 97-106.

SIMON Gildas (1995) Géodynamique des migrations internationales dans le monde, Paris, PUF, Coll. Politique d'Aujourd'hui, 429 p.

SCHWAB R. (1971) Les champs migratoires, critères d'étude de la genèse et de l'évolution des structures régionales d'un espace. Bulletin de l'Association des Géographes français, n³93-394, pp. 369-375.

TAPIA, Stéphane de (1998) La circulation migratoire dans les diasporas ouvrières de la Méditerranée. Turquie Maroc. Rapport final d'une recherche financée par le Ministère de l'éducation nationale et de la culture, Poitiers, MIGRINTER, 2 vol., 224 p.

TARRIUS Alain (1996) Territoires circulatoires des migrants et espaces européens. M. Hirschorn et J -M. Berthelot (dir.), Mobilités et ancrages, Paris, L'Harmattan.

TARRIUS Alain (1992) Les fourmis d'Europe. Migrants riches, migrants pauvres et nouvelles villes internationales, Editions de l'Aube, 265 p. 